\title{
Reversal of Medetomidine-Ketamine Combination Anesthesia in Rabbits by Atipamezole
}

\author{
Min Su KIM1), Seong Mok J EONG'1), J ae Hak PARK ${ }^{2)}$, \\ Tchi Chou NAM ${ }^{1)}$ and Kang Moon SEO')
}

\author{
1)Department of Veterinary Surgery, and ${ }^{2}$ Department of Laboratory Animal Medicine, \\ College of Veterinary Medicine, Seoul National University, San 56-1, Shillim \\ 9-dong, Kwanak-gu, Seoul, 151-742, Korea
}

\begin{abstract}
This study was performed to determine the optimal reversal dosage of atipamezole on medetomidine-ketamine combination anesthesia. The subject rabbits were divided into five groups ( $n=5 /$ group), and all were anesthetized with intravenous medetomidine $(0.35 \mathrm{mg} / \mathrm{kg})$ and ketamine $(5 \mathrm{mg} / \mathrm{kg})$. Atipamezole was administered intravenously 35 min after administration of the medetomidine-ketamine mixture, at doses of a quarter, a half, equal, or two times higher than the preceding medetomidine-ketamine dose according to experimental group. Heart rate (HR), mean arterial pressure (MAP), respiratory rate $(R R)$ and rectal temperature $(R T)$ were measured every five minutes and the mean arousal time (MAT) was also recorded. This study revealed that the optimal atipamezole dosage to achieve reversal effects is equal to or double the dose of medetomidine. At these dosages, $H R$ and MAP significantly recovered and MAT was significantly shortened with no side effects being observed $(p<0.05)$.
\end{abstract}

Key words: anesthesia, atipamezole, ketamine, medetomidine, rabbit

\section{Introduction}

Physical or chemical restraint of laboratory animals is often required for a variety of experimental protocols, including minor and short-term procedures [3]. Rabbits are difficult to anesthetize, and show a wide range of responses [12]. There are many anesthetic methods for rabbits that use a combination of several injection delivered drugs [4]. The xylazine-ketamine combination is an effective protocol for rabbit anesthesia [24], due to its low cost and ease of administration [11, 15]. However, this combination has been reported to have some mortality [24] and fatal responses in spite of inducing good anesthesia in rabbits [2, 14]. Among the alpha-2 adrenoceptor agonists, medetomidine is known as a potent and selective alpha-2 adrenoceptor agonist with both sedative and analgesic effects [16]. Medetomidineketamine combination anesthesia is widely used in veterinary clinics [5, 9] and has recently been used in animal laboratories [12, 17]. This combination has proved to be a very useful chemical restraint, producing light anesthesia and good immobilization [3]. However,

(Received 14 June 2004 / Accepted 15 June 2004)

Address corresponding: K.M. Seo, Department of Veterinary Surgery, College of Veterinary Medicine, Seoul National University, San 56-1, Shillim 9-dong, Kwanak-gu, Seoul, 151-742, Korea 
medetomidine may produce hypotension, bradycardia and cardiac arrhythmia in many species, especially following intravenous administration [16]. These side effects of medetomidine can be quickly reversed by atipamezole, 4-(2-ethyl-2,3-dihydro-1H-inden-2-yl)-1H-imidazole $\mathrm{HCl}$, the most recent and specific alpha-2 antagonist [3, 23]. However, the suitable dosage of intravenous atipamezole for the rabbit has not been reported yet. This study was conducted to evaluate the optimal dosage of atipamezole for a fast reverse effect and minimum side effects on medetomidine-ketamine anesthesia in rabbits.

\section{Materials and Methods}

\section{Animals}

Twenty-five young adult conventional New Zealand White rabbits (Oryctolagus cunniculus) of both sexes with an average body weight of $3 \mathrm{~kg}$ ( $2.50 \mathrm{~kg}$ to 3.50 $\mathrm{kg}$ ) were used in these experiments. A commercial pellet diet (Purina pellet, Purina Korea) and water were supplied ad libitum, but food was withheld for $12 \mathrm{~h}$ prior to each experiment. The rabbits were housed in individual stainless steel cages in a controlled environment, at temperatures of $20-25^{\circ} \mathrm{C}$ with a $12 \mathrm{~h}$ light $/ 12 \mathrm{~h}$ dark cycle per day. The experiment adhered to the strict guidelines of the "Guide for the Care and Use of Laboratory Animals" of Seoul National University (Seoul, South Korea).

\section{Anesthesia}

Medetomidine (DOMITOR ${ }^{\circledR}$ Orion Animal Health/ Turku, Finland), atipamezole (ANTISEDAN ${ }^{\circledR}$ Orion Animal Health/Turku, Finland) and ketamine (KEIRAN ${ }^{\circledR}$ Korea United Pharm/Korea) were used in this study. Normal physiological saline (DAIHAN sterile $\mathrm{NaCl}^{\circledR}$ Daihan Pharm/Korea) was used for dilution. Heart rate (HR), mean arterial pressure (MAP), respiratory rate (RR) and rectal temperature (RT) were simultaneously recorded using an anesthetic patient monitoring system (S-5, Datex-Ohmega, Helsinki, Finland). An intravenous catheter (D\&B-Cath ${ }^{\circledR}$, Sin Dong Bang Medial Co., Seoul, Korea) was placed into the ear vein for the administration of medetomidine, ketamine, atipamezole and maintenance fluid (normal saline $0.9 \%$ ). A $22 \mathrm{G}, 4 \mathrm{~cm}$ needle was inserted into the femoral artery and the catheter was connected to a calibrated pressure transducer (TranStar ${ }^{\circledR}$ Single Monitoring
Kit, MX9504, A Furon Compony, Hilliard, USA) and also to the anesthetic patient monitoring system.

\section{Experimental procedure}

Each rabbit was rested for $30 \mathrm{~min}$ after instrumentation for stabilization. Baseline parameters were measured $5 \mathrm{~min}$ before the administration of anesthetics. Medetomidine was administered intravenously via the left ear vein at a dose of $0.35 \mathrm{mg} / \mathrm{kg}$, followed by 5 $\mathrm{mg} / \mathrm{kg}$ ketamine intravenously after an interval of 5 min, as previously described [8]. Pure oxygen was delivered to the animal's nose through a face mask throughout the experimental period. Thirty-five minutes after the medetomidine-ketamine administration, atipamezole was administered intravenously at doses of $0.09 \mathrm{mg} / \mathrm{kg}$ (group B, n=5), $0.18 \mathrm{mg} / \mathrm{kg}$ (group C, $\mathrm{n}=5$ ), $0.35 \mathrm{mg} / \mathrm{kg}$ (group $\mathrm{D}, \mathrm{n}=5$ ) or $0.70 \mathrm{mg} / \mathrm{kg}$ (group $\mathrm{E}$, $\mathrm{n}=5$ ). These doses were a quarter, a half, equal to, and double the proceeding dose of medetomidine for groups B,C,D and E, respectively. The total volume of each atipamezole administration was adjusted to the same volume of group E. For the control (group A, n=5), an equal amount of normal saline $(0.35 \mathrm{mg} / \mathrm{kg})$ was administered. Arousal times (AT) the period from the time of administration of atipamezole to the time of showing the first signs of the righting response were measured and the mean arousal time (MAT) was determined. Heart rate, MAP, respiratory rate and rectal temperature were measured for a total of 65 min (Fig. 1).

\section{Statistical analysis}

Data were analyzed using the SPSS 10.0 analysis program. To compare the cardiopulmonary parameters between baseline and post-anesthesia in each group, Wilcoxon's signed rank test was used $(\mathrm{p}<0.05)$. For comparison of cardiopulmonary parameters and MAT among groups, the Kruskal-Wallis test was used $(\mathrm{p}<0.05)$.

\section{Results}

This study found that at dosages of $0.35 \mathrm{mg} / \mathrm{kg}$ or $0.7 \mathrm{mg} / \mathrm{kg}$, intravenous atipamezole effectively reversed the medetomidine-ketamine combinations anesthesia in the rabbit with no apparent side effects.

Mean arousal times (MAT) were shown to be significantly dose-dependent in this study $(\mathrm{p}<0.05)$. While 


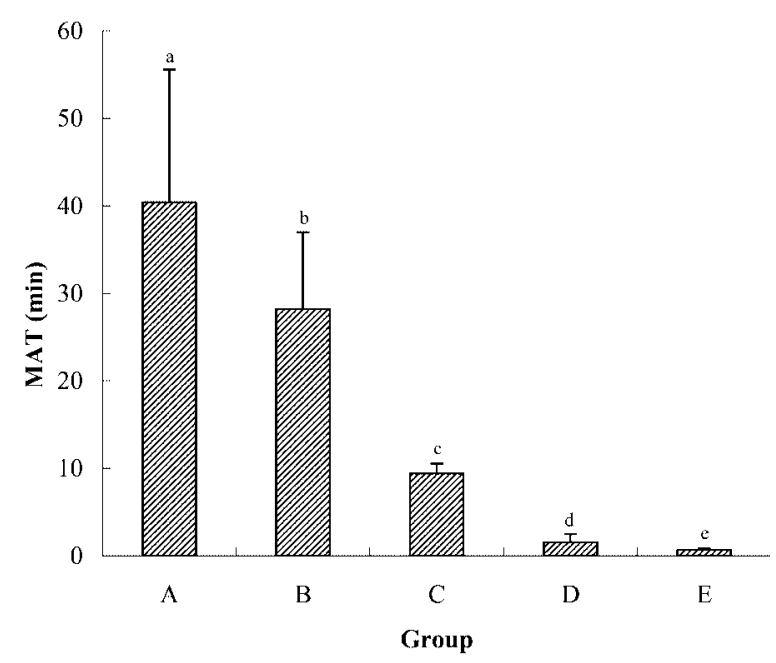

Fig. 1. Effect of atipamezole on mean arousal time (MAT) in rabbits anesthetized with medetomidine-ketamine. Arousal time was defined as the time between administration of atipamezole to the first signs of a righting reflex. Group A: control. Group B: atipamezole 0.09 $\mathrm{mg} / \mathrm{kg}$. Group C: atipamezole $0.18 \mathrm{mg} / \mathrm{kg}$. Group D: atipamezole $0.35 \mathrm{mg} / \mathrm{kg}$. Group E: atipamezole 0.7 $\mathrm{mg} / \mathrm{kg}$. a, b, c, d and e denote significant differences from groups A, B, C, D and E, respectively; MannWhitney $U$ test $(\mathrm{p}<0.05)$.

the MAT of the control group was $40.4 \pm 15.2 \mathrm{~min}$, those of the treated groups (group B, C, D and E) were $28.2 \pm 8.8 \mathrm{~min}, 9.4 \pm 1.14 \mathrm{~min}, 1.55 \pm 0.94 \mathrm{~min}$, and $0.68 \pm 0.16 \mathrm{~min}$, respectively (Fig. 1).

Heart rate significantly decreased immediately after administration of medetomidine in all groups $(\mathrm{p}<0.05)$. In groups $\mathrm{A}, \mathrm{B}$ and $\mathrm{C}$, heart rates had still not fully recovered $20 \mathrm{~min}$ after administration of the saline or atipamezole. In group D and $\mathrm{E}$ however, there was a rapid recovery after administration of atipamezole $(\mathrm{p}<0.05$, Fig. 2).

Mean arterial pressure (MAP) was likewise significantly reduced after medetomidine administration in all groups, but was significantly raised after atipamezole administration in groups D and $\mathrm{E}$ ( $\mathrm{p}<0.05$, Fig. 3).

Respiratory rate was also significantly reduced after administration of medetomidine $(\mathrm{p}<0.05)$, but increased after administration of ketamine in all groups. In groups $\mathrm{A}$ and $\mathrm{B}$, respiratory rates had not recovered $20 \mathrm{~min}$ after administration of saline or low dose of atipamezole, whereas groups $\mathrm{C}, \mathrm{D}$ and $\mathrm{E}$ experienced significant recoveries after administration of the higher doses of atipamezole ( $<<0.05$, Fig. 4).

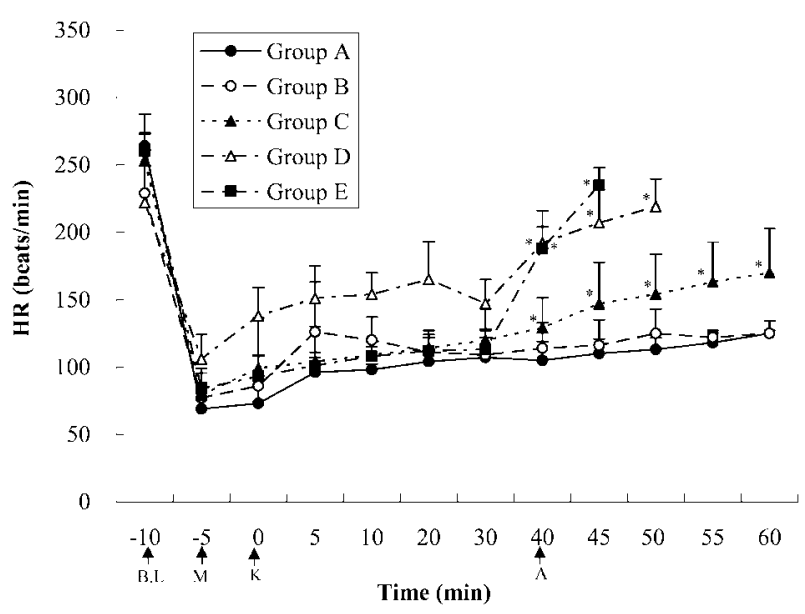

Fig. 2. Changes in heart rate(HR) after administration of atipamezole in medetomidine-ketamine anesthetized rabbits $(n=5)$. Group A: control. Group B: atipamezole 0.09 $\mathrm{mg} / \mathrm{kg}$. Group C: atipamezole $0.18 \mathrm{mg} / \mathrm{kg}$. Group D: atipamezole $0.35 \mathrm{mg} / \mathrm{kg}$. Group E: atipamezole $0.7 \mathrm{mg} /$ $\mathrm{kg}$. B.L: baseline. M: immediately after administration of medetomidine. K: immediately after administration of ketamine. A: immediately after administration of atipamezole. *: Significant difference after administration of atipamezole in HR within group. Wilcoxon's signed rank test $(\mathrm{p}<0.05)$.

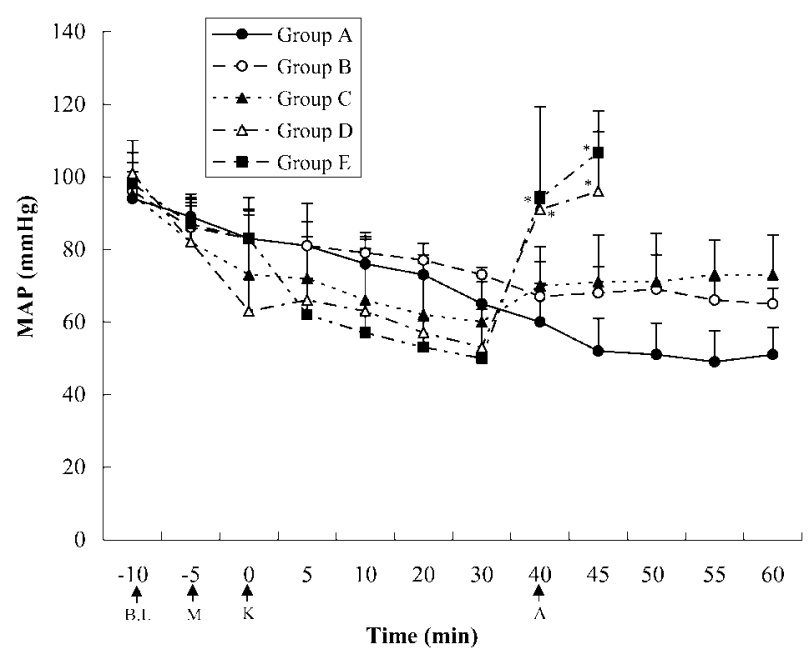

Fig. 3. Changes in mean arterial pressure (MAP) after administration of atipamezole in medetomidine-ketamine anesthetized rabbits $(n=5)$. Group A: control. Group B: atipamezole $0.09 \mathrm{mg} / \mathrm{kg}$. Group C: atipamezole $0.18 \mathrm{mg} /$ $\mathrm{kg}$. Group D: atipamezole $0.35 \mathrm{mg} / \mathrm{kg}$. Group E: atipamezole $0.7 \mathrm{mg} / \mathrm{kg}$. B.L: baseline. M: immediately after administration of medetomidine. K: immediately after administration of ketamine. A: immediately after administration of atipamezole. *: Significant difference after administration of atipamezole in MAP within group; Wilcoxon's signed rank test $(\mathrm{p}<0.05)$. 


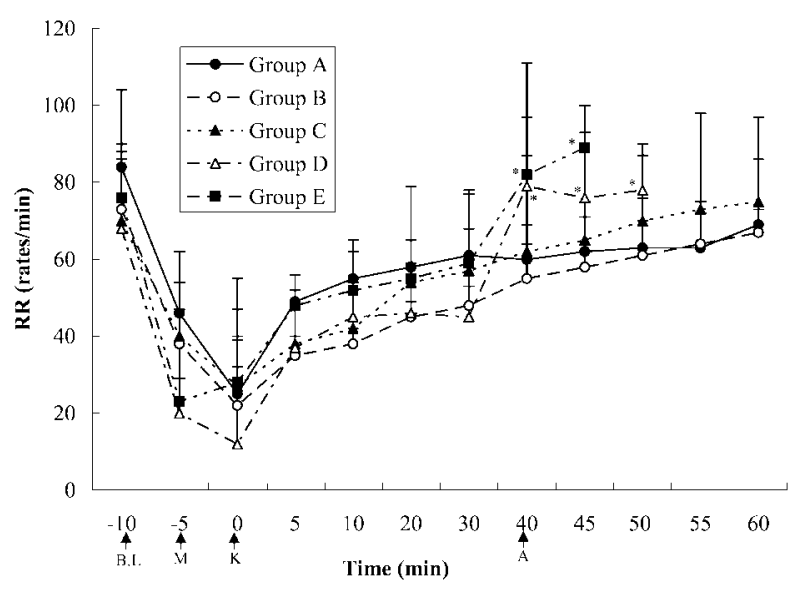

Fig. 4. Changes in respiratory rate after administration of atipamezole in medetomidine-ketamine anesthetized rabbits $(n=5)$. Group A: control. Group B: atipamezole $0.09 \mathrm{mg} / \mathrm{kg}$. Group C: atipamezole $0.18 \mathrm{mg} / \mathrm{kg}$. Group D: atipamezole $0.35 \mathrm{mg} / \mathrm{kg}$. Group E: atipamezole 0.7 $\mathrm{mg} / \mathrm{kg}$. B.L: baseline. M: immediately after administration of medetomidine. K: immediately after administration of ketamine. A: immediately after administration of atipamezole. *: Significant difference after administration of atipamezole in MAP within group; Wilcoxon's signed rank test $(\mathrm{p}<0.05)$.

Rectal temperatures in all groups gradually decreased with time (Fig. 5).

\section{Discussion}

Various dose combinations of medetomidineketamine in rabbits and their anesthetic duration have been reported $[4,12]$. Usually short-term anesthesia is needed for clinical procedures involving rabbits. It was reported that the administration of $0.35 \mathrm{mg} / \mathrm{kg}$ medetomidine (intramuscular) followed by $5 \mathrm{mg} / \mathrm{kg}$ ketamine (intravenous) provides an adequate anesthetic depth together with no side effects and zero mortality [8]. In our study $0.35 \mathrm{mg} / \mathrm{kg}$ of intravenous medetomidine induced moderate sedation, and anesthesia was satisfactorily maintained with $5 \mathrm{mg} / \mathrm{kg}$ of intravenous ketamine. All rabbits were immobilized and showed good muscle relaxation with no side effects. The administration of the antidote, atipamezole, reduced the remnant anesthetic maintenance time and avoided the side effects described before.

Atipamezole is a known potent and highly selective specific alpha-2 adrenoceptor antagonist which produces

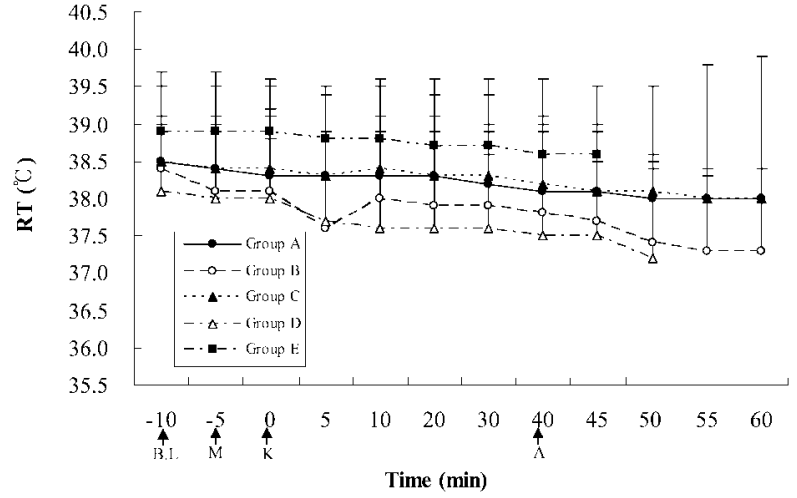

Fig. 5. Changes in rectal temperature after administration of atipamezole in medetomidine-ketamine anesthetized rabbits $(n=5)$. Group A: control. Group B: atipamezole $0.09 \mathrm{mg} / \mathrm{kg}$. Group C: atipamezole $0.18 \mathrm{mg} / \mathrm{kg}$. Group D: atipamezole $0.35 \mathrm{mg} / \mathrm{kg}$. Group E: atipamezole 0.7 $\mathrm{mg} / \mathrm{kg}$. B.L: baseline. M: immediately after administration of medetomidine. K: immediately after administration of ketamine. A: immediately after administration of atipamezole.

effective antagonism of anesthesia induced by medetomidine-ketamine [4, 17].

In the present study, anesthetics were administered intravenously for rapid induction and short-term duration. Anesthesia was rapidly induced immediately after intravenous administration of ketamine. In the control group, the anesthetic state was maintained for $40.4 \pm$ $15.2 \mathrm{~min}$. In all atipamezole treated groups, MAT was significantly reduced especially in the groups treated with doses of $0.35 \mathrm{mg} / \mathrm{kg}$ and $0.7 \mathrm{mg} / \mathrm{kg}$ which showed MATs of under 2 min.

Medetomidine can induce bradycardia, brady arrhythmias, respiratory depression, and hypotension [1, 16, 19]. Bradycardia and sinus arrhythmia are related to changes in blood pressure [9]. It is possible that the central alpha-2 adrenoceptor mediated hypotension is partly counteracted by the concomitant stimulation of the peripheral vasoconstrictive postsynaptic alpha-2 adrenoceptors, which is reflected in the initial hypertension phase following drug administration $[16,19]$. Atipamezole, an alpha-2 adrenergic antagonist, is able to antagonize the cardiovascular effects of the anesthetic [1, 22].

Heart rate sharply decreases after intravenous administration of medetomidine then mildly increases after intravenous administration of ketamine [25]. In our 
study, it recovered 5 min after administration of atipamezole in groups C, D and E. Hypotension was observed after administration of medetomidine, and it recovered in a similar manner to the baseline value found in groups $\mathrm{D}$ and $\mathrm{E}$.

In this study, respiratory rate was lowered after the administration of medetomidine. Although the respiratory rate was mildly irregular after intravenous administration of ketamine [6,7], it remarkably increased after administration of atipamezole in groups D and E. Rectal temperature was continuously decreased after the intravenous administration of atipamezole in all groups. The temperature effects of medetomidineketamine anesthesia that have been reported show various responses to the administration of atipamezole $[21,26]$. In this study, no relationship was found between increased rectal temperature and the administration of atipamezole.

For many species the optimal dosage of atipamezole to reverse the anesthetic effects of medetomidine has been studied and determined. In the laboratory beagle, the optimal dose of atipamezole is 4 to 10 times the proceeding dose of medetomidine [20]. For horses, atipamezole at doses 8 to 12 times larger are able to reverse the effects of medetomidine [13]. In this study using rabbits, an intravenous atipamezole dose of 1 to 2 times the preceding doses of medetomidine was found to safely reverse the anesthetic effects of intravenous medetomidine-ketamine anesthesia with no side effects. The reason different doses of atipamezole are needed among different species has not yet been determined but Jalanka reported that there might be related differences in regulatory mechanisms mediated by alpha- 2 adrenoceptors [10].

In conclusion, medetomidine-ketamine is a suitable combination for short-term procedure anesthesia in rabbits. Atipamezole has been shown to be an effective antagonist of medetomidine-ketamine combinations for clinical use in rabbits and this study determined the optimal dosage.

Mean arousal times (MAT) following the administration of atipamezole were shown to significantly decrease dose dependently $(\mathrm{p}<0.05)$. Heart rate and MAP both recovered effectively after administration doses equal to or double the initial dose of medetomidine $(\mathrm{p}<0.05)$. Thus the suitable dosage of atipamezole producing a safe and effective reversal for rabbits was determined to be 1 to 2 times higher than the administered dose of medetimidine.

\section{References}

1. Bergström, K. 1988. Cardiovascular and pulmonary effects of a new sedative/analgesic(medetomidine) as a preanesthetic drug in the dog. Acta Vet. Scand. 29: 109-116.

2. Beyers, T.M., Richardson, J.A., and Prince, M.D. 1991. Axonal degeneration and self-mutilation as a complication of the intramuscular use of ketamine and xylazine in rabbits. Lab. Anim. Sci. 41: 519-520.

3. Cruz, J.I., Loste, J.M., and Burzaco, O.H. 1998. Observations on the use of medetomidine/ketamine and its reversal with atipamezole for chemical restraint in the mouse. Lab. Anim. 32: 18-22.

4. Flecknell, P.A. 2000. Anaesthesia. pp. 105-107. In: Manual of Rabbit Medicine and Surgery, British Small Animal Veterinary Association, Hampshire.

5. Fleiss, J.L. 1986. The Design and Analysis of Clinical Experiments, John Wiley \& Sons, New York.

6. Hatch, R.C. 1973. Prevention of ketamine catalepsy and enhancement of ketamine anesthesia in cats pretreated with methiothepin. Pharmacol. Res. Commun. 5: 311-319.

7. Hatch, R.C. and Ruch, T. 1974. Experiments on antagonism of ketamine anesthesia in cats given adrenergic, serotonergic and cholinergic stimulants alone and in combination. Am. J. Vet. Res. 35: 35-39.

8. Hellebrekers, L.J., de Boer, E.J., van Zuylen, M.A., and Vosmeer, H. 1997. A comparison between medetomidineketamine and medetomidine-propofol anesthesia in rabbits. Lab. Anim. 31: 58-69.

9. Jalanka, H. 1989. The use of medetomidine, medetomidineketamine combinations and atipamezole at Helsinki zoo a review of 240 cases. Acta Vet. Scand. Suppl. 85: 193-197.

10. Jalanka, H. 1990. Medetomidine- and medetomidineketamine-induced immobilization in blue foxes (Alopex lagopus) and its reversal by atipamezole. Acta Vet. Scand. 31: 63-71.

11. Lipman, N.S., Phillips, P.A., and Newcomer, C.E. 1987. Reversal of ketamine/xylazine anesthesia in the rabbit with yohimbine. Lab. Anim. Sci. 37: 474-477.

12. Nevalainen, T., Pyhala, L., Voipio, H.M., and Virtanen, R. 1989. Evaluation of anesthetic potency of medetomidineketamine combination in rats, guinea-pigs and rabbit. Acta Vet. Scand. Suppl. 85: 139-143.

13. Nilsfors, L. and Kvart, C. 1986. Preliminary report on the cardiorespiratory effects of the antagonist to detomidine, MPV-1248. Acta Vet. Scand. Suppl. 82: 121-129.

14. Palmore, W.P. 1990. A fatal response to xylazine and ketamine in a group of rabbits. Vet. Res. Commun. 14: 9198.

15. Sanford, T.D. and Colby, E.D. 1980. Effect of xylazine and ketamine on blood pressure, heart rate and respiratory rate in rabbits. Lab. Anim. Sci. 30: 519-523.

16. Savola, J.M. 1989. Cardiovascular actions of medetomidine and their reversal by atipamezole. Acta Vet. Scand. Suppl. 
85: 39-47.

17. Thurmon, J.C., Tranquilli W.J., and Benson G.J. 1996. Lumb \& Johnes' Veterinary Anesthesia, 3th ed., Lippincott Williams \& Wilkins, Philadelphia.

18. Vähä-Vahe, T. 1989. Clinical evaluation of medetomidine, a novel sedative and analgesic drug for dogs and cats. Acta Vet. Scand. 30: 267-273.

19. Vainio, O. 1989. Introduction to the clinical pharmacology of medetomidine. Acta Vet. Scand. Suppl. 85: 85-88.

20. Vainio, O. and Vähä-Vahe, T. 1990. Reversal of medetomidine sedation by atipamezole in dogs. J. Vet. Pharmacol. Ther. 13: 15-22.

21. Verstegen, J., Fargetton, X., Zanker, S., Donnay, I., and Ectors, F. 1991. Antagonistic activities of atipamezole, 4aminopyridine and yohimbine against medetomidine/ ketamine-induced anesthesia in cats. Vet. Rec. 128: 57-60.

22. Virtanen, R. 1989. Pharmacological profiles of medetomidine and its antagonist, atipamezole. Acta Vet Scand. Suppl. 85: 29-37.

23. Virtanen, R., Savola, J.M., and Saano, V. 1989. Highly selective and specific antagonism of central and peripheral alpha-2 adrenoceptors by atipamezole. Arch. Int. Pharmacodyn. Ther. 297: 190-204.

24. White, G.L. and Holmes, D.D. 1976. A comparison of ketamine and the combination ketamine-xylazine for effective surgical anesthesia in the rabbit. Lab. Anim. Sci. 26: 804-806.

25. Wright, M. 1982. Pharmacologic effects of ketamine and its use in veterinary medicine. J. Am. Vet. Med. Assoc. 180: 1462-1471.

26. Yamashita, K., Yonezawa, K., Izumisawa, Y., and Kotani, T. 1996. Antagonistic effects of atipamezole medetomidineinduced sedation in horses. J. Vet. Med. Sci. 58: 1049-1052. 